\title{
PAPERS
}

\section{Effects of a modified live CSFV vaccine on the development of PMWS in pigs infected experimentally with PCV-2}

\author{
Y. Ha, E-M. Lee, Y. H. Lee, C. H. Kim, D. Kim, S. Chae, K. K. Ahn, B. Kim, C. Chae
}

The objective of this study was to determine the effect of vaccination against classical swine fever virus (CSFV) on the development of postweaning multisystemic wasting syndrome (PMWS) in conventional pigs infected experimentally with porcine circovirus type 2 (PCV-2). The pigs infected with PCV-2 and immunised with modified live CSFV developed mild to moderate PMWS, whereas none of the pigs infected with PCV-2 alone or immunised with modified live CSFV alone developed PMWS. Lesions histologically characteristic of PMWS were observed in lymph nodes from the pigs infected with PCV-2 and immunised with modified live CSFV vaccine, and extensive replication of $P C V-2$ was detected in the nodes by in situ hybridisation.

PORCINE circovirus type 2 (PCV-2), a single-stranded DNA virus in the family Circoviridae, is recognised as the aetiological agent of postweaning multisystemic wasting syndrome (PMWS) (Allan and Ellis 2000, Chae 2004). Although PCV-2 is the major aetiological agent of PMWS in pigs, experimental infection of pigs with PCV-2 alone produces only a mild infection without clinical signs and modest lymphadenopathy (Krakowka and others 2000). In order to produce full PMWS in pigs, they must be infected with PCV-2 and porcine parvovirus (PPV) or PCV-2 plus immunostimulation (Allan and others 1999, Krakowka and others 2001, Kim and others 2003). Moreover, in field and laboratory studies, the use of certain commercial swine bacterins has been associated with an increased incidence of PMWS in the vaccinated animals (Allan and others 2001, Kyriakis and others 2002, Opriessnig and others

Veterinary Record (2009) 164, 48-51

Y. Ha, DVM,

E-M. Lee,

Y. H. Lee, DVM,

C. H. Kim, DVM,

D. Kim, DVM,

S. Chae, DVM

K. K. Ahn, DVM, MS,

B. Kim, DVM, MS,

C. Chae, DVM, MS, PhD,

Seoul National University,

College of Veterinary Medicine,

Department of Veterinary Pathology,

San 56-1, Shillim-Dong,

Gwanak-Gu, Seoul, 151-742,

Republic of Korea
2003, Krakowka and others 2007). However, the swine vaccine had no potentiating effect on the replication of PCV-2 or on the development of PMWS (Resendes and others 2004).

Classical swine fever virus (CSFV) is the causative agent of classical swine fever, otherwise known as hog cholera, a devastating disease of swine with economically serious consequences. For this reason, many countries pursue surveillance and/or eradication programmes to limit the disease (Moennig 1992). In Korea, CSF was reported in Kyounggi Province in August 1999, and a major turning point in dealing with the disease was reached in December 2001, when the country committed to a stamping-out policy without relying on vaccines to control it. However, several outbreaks were reported in Kangwon and Kyounggi Provinces between April and October 2002, and from March 2003 the National Veterinary Research and Quarantine Service returned to the use of the modified live CSFV vaccine.

During 2003 and 2004, tissues from 446 postweaning pigs were examined and PMWS was diagnosed in 187 (41.9 per cent) of them, a two-fold increase over its incidence in 2002, when the modified live CSFV vaccine had not been used (C. Chae, personal observation). Furthermore, many swine practitioners and producers suspected that the use of the modified live vaccine had potentiated the induction of PMWS in the field. The objective of this study was to determine whether vaccination of conventional pigs against CSFV would enhance the incidence and severity of PCV-2 associated lesions of PMWS in them.

\section{Materials and methods}

\section{Experimental design}

Tissue culture-propagated PCV-2, strain SNUVR000463 (second passage), and porcine parvovirus (PPV), strain SNUVR000464 (second passage), were used as the sources of the viral inocula (Kim and others 2003). For the inoculations, a PCV-2 pool containing $1.2 \times 10^{5}$ TCID50/ $\mathrm{ml}$ and a PPV pool containing $1.3 \times 10^{5} \mathrm{TCID} 50 / \mathrm{ml}$ were prepared as described by Krakowka and others (2000). Thirty conventional 20-dayold pigs, all of which were seronegative for PCV, PPV, and porcine reproductive and respiratory syndrome virus (PRRSV), were divided randomly into five groups (Table 1 ).

The six pigs in group 1 were inoculated intranasally with $1 \mathrm{ml}$ of a 1:20 dilution of the PCV-2 pool at 24 days of age; the six pigs in group 2 were inoculated intranasally with $1 \mathrm{ml}$ of a 1:20 dilution of the PCV-2 pool at 24 days of age, followed by intramuscular injections of modified live CSFV vaccine in the thigh at 40 and 60 days of age; the six pigs in group 3 were inoculated intranasally with $2 \mathrm{ml}$ of a mixture of equal volumes of the 1:20 dilutions of the PCV-2 pool and the PPV pool; the six pigs in group 4 were inoculated intranasally with lysates of PCV-free PK-15 cells and then injected intramuscularly in the thigh with modified live CSFV vaccine at 40 and 60 days of age as vaccinated negative controls; the six pigs in group 5 were inoculated intranasally with lysates of PCV-free PK-15 cells as negative controls. The five groups of pigs were housed separately in isolators (three pigs per isolator) and examined at regular intervals. 
TABLE I: Treatments given to five groups of six pigs

\begin{tabular}{lccccc} 
Treatment & Group & \multicolumn{5}{c}{. } & 2 & 3 & 4 & 5 \\
PCV-2 infection & + & + & + & - & - \\
PPV infection & - & - & + & - & - \\
CSFV vaccination & - & + & - & + &
\end{tabular}

CSFV Modified live classical swine fever virus, PCV-2 Porcine circovirus type 2, PPV Porcine parvovirus

They were weighed when they were $28,32,35,39,42,46,49,53,60$, 63, 67 and 70 days of age. Three of the pigs in each group were euthanased at 42 days after inoculation and examined postmortem. The pigs were sedated by an intravenous injection of sodium pentobarbital and then euthanased by electrocution (Anon 2001). Superficial inguinal lymph nodes, which have been found to show consistent and intense labelling for PCV-2 and typical granulomatous lesions (Kim and others 2003) were collected and fixed in 10 per cent $(\mathrm{v} / \mathrm{v})$ phosphate-buffered formalin for one to two days before being processed for histological examination. The methods were approved by the Seoul National University Institutional Animal Care and Use Committee.

\section{In situ hybridisation}

To develop the probe for in situ hybridisation, PCR products of PCV-2 were purified by using a membrane filter with a $30 \mathrm{kDa}$ cut-off. The nucleotide sequences of the purified PCR products were determined by means of BigDye chemistry with the ABI Prism Sequencer (Applied Biosystems). The purified PCR products were sequenced before they were labelled by random priming with digoxigenin-dUTP (Boehringer Mannheim) according to the manufacturer's instructions. In situ hybridisation for PCV-2 and PPV was carried out as described by Kim and others (2003).

\section{Lesion score}

The lesions in the lymph nodes were scored according to the estimated amount of granulomatous inflammation and lymphoid depletion of follicles, as described by Kim and Chae (2004). The scores ranged from 0
TABLE 2: Number of pigs in each group of six pigs that were affected by the different types of histological lesions

\begin{tabular}{|c|c|c|c|c|c|}
\hline \multirow[b]{2}{*}{ Type of lesion and site } & \multicolumn{5}{|c|}{ Group } \\
\hline & 1 & 2 & 3 & 4 & 5 \\
\hline \multicolumn{6}{|l|}{ Granulomatous lesion } \\
\hline Lymph node & 0 & 6 & 6 & 0 & 0 \\
\hline Peyer's patches & 0 & 3 & 3 & 0 & 0 \\
\hline Tonsil & 0 & 1 & 2 & 0 & 0 \\
\hline Spleen & 0 & 3 & 2 & 0 & 0 \\
\hline \multicolumn{6}{|l|}{ Lymphoid depletion } \\
\hline Lymph node & 2 & 6 & 6 & 0 & 0 \\
\hline Peyer's patches & 0 & 4 & 3 & 0 & 0 \\
\hline Tonsil & I & 1 & 1 & 0 & 0 \\
\hline Spleen & 1 & 3 & 4 & 0 & 0 \\
\hline
\end{tabular}

Normal, no signs of lymphoid depletion or granulomatous replacement to 5 Severe signs.

\section{Morphometric analysis}

Single sections from each formalin-fixed superficial inguinal lymph node from the pigs in groups 1, 2 and 3 were taken for morphometric analysis as described by Kim and others (2003). To obtain quantitative data, the morphometric analysis of the in situ hybridisation slides was carried out using the NIH Image J Program (National Institutes of Health). In each case, three fields were selected randomly, the numbers of positive cells per unit area $\left(0.25 \mathrm{~mm}^{2}\right)$ were counted, and the mean values were calculated.

\section{Statistical analysis}

The data were analysed using Prism4 software (GraphPad Software). One-way analysis of variance and Tukey's test were used to compare the mean bodyweights of the five groups. The Kruskall-Wallis one-way nonparametric analysis of variance for more than two independent groups and the Mann-Whitney $U$ test (for two independent groups) were used to compare the numbers of PCV-2-positive cells among the three infected groups, the Student's $t$ test was used to compare the lesion scores of groups 2 and 3. Statistical significance was accepted at $\mathrm{P}<0.05$.

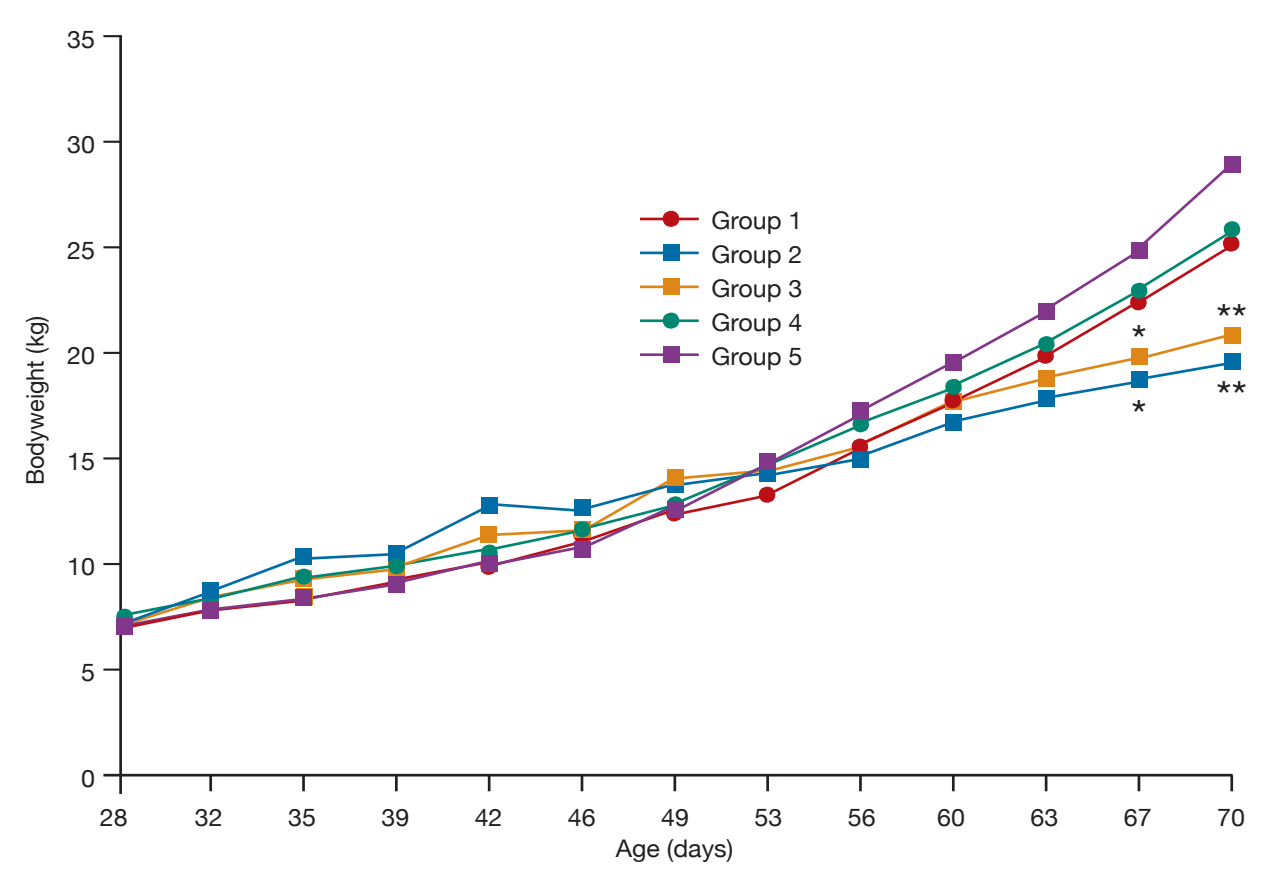

FIG I: Mean bodyweight of the groups of six pigs infected with porcine circovirus type 2 (PCV-2) (group I), infected with PCV-2 and immunised with modified live classical swine fever virus (CSFV) vaccine (group 2), co-infected with PCV-2 and porcine parvovirus (PPV) (group 3), immunised with modified live CSFV vaccine alone (group 4), and the negative control pigs (group 5). The mean bodyweight of the pigs in group 5 increased significantly in comparison with the other groups. * $\mathbf{P}<0.05$, ** $\mathbf{P}<0.001$

\section{Results}

\section{Clinical signs}

Clinical signs of PMWS were observed only in groups 2 and 3 . The group 2 pigs (PCV-2 plus CSFV vaccine) became lethargic 22 days after they were inoculated and they all appeared to be thin after 36 days, when they were 60 days old. The pigs in group 3 (PCV-2 and PPV) appeared thin 29 days after they were inoculated, when they were 63 days old. The pigs in groups 1, 4 and 5 remained clinically normal.

\section{Bodyweight}

There were no significant differences in the mean bodyweights of the five groups between 28 and 63 days of age. The mean bodyweight of the group 5 pigs, the negative controls, became significantly greater than those of the group 2 pigs (PCV-2 plus CSFV vaccine) and group 3 pigs (PCV-2 plus $P P V)$ at $67(\mathrm{P}<0.05)$ and $70(\mathrm{P}<0.001)$ days of age (Fig 1$)$.

The mean bodyweight of the pigs in group 2 (PCV-2 plus CSFV vaccine) was lower than that of the pigs in group 4 (CSFV vaccine only $)$ at $67(\mathrm{P}<0.05)$ and $70(\mathrm{P}<0.05)$ days of age. The mean bodyweight of the pigs in group 3 (PCV-2 plus PPV) was significantly lower than that of the pigs in group 4 (CSFV vaccine only) at 70 days of age $(\mathrm{P}<0.05)$. The pigs in group 2 (PCV-2 plus CSFV vaccine) 


FIG 2: Cluster of
histiocytes and
multinucleated
giant cells in an
inguinal lymph node
derived from a pig
infected with porcine
circovirus type 2
and immunised with
modified live classical
swine fever virus
vaccine (group 2 ) and
euthanased 42 days
later. Haematoxylin
and eosin. $x 200$

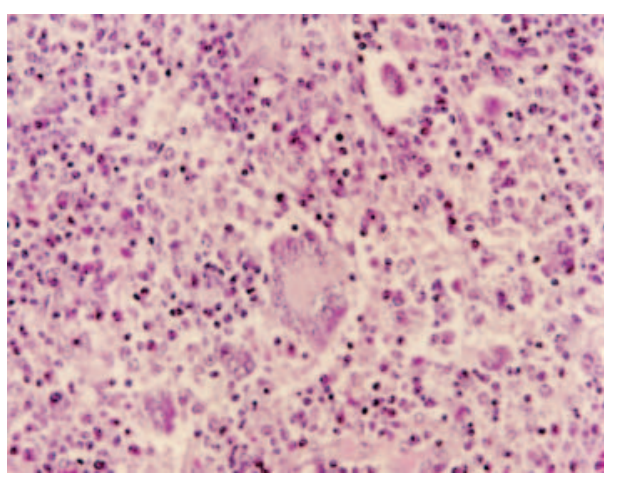

weighed significantly less than the pigs in group 3 (PCV-2 plus PPV) and group 1 (PCV-2 only) at 70 days of age $(\mathrm{P}<0.05)$.

\section{Macroscopic and microscopic lesions}

When they were examined postmortem 42 days after inoculation, enlarged and pale tracheobronchial, mesenteric and mediastinal lymph nodes were observed in the pigs from group 2 ( $\mathrm{PCV}-2$ plus CSFV vaccine) and group 3 (PCV-2 and PPV). No gross lesions were detected in the pigs from groups 1, 4 and 5. Characteristic histological lesions were observed mainly in the lymphoid tissues from the pigs in groups 2 and 3 and occasionally in the lymphoid tissues from the pigs in group 1 (PCV-2 only). No histopathological changes were observed in the pigs from groups 4 and 5 (Table 2). The most striking lesions consisted of granulomatous inflammation and lymphoid depletion in the lymph nodes, ileal Peyer's patches, tonsil and spleen. The multifocal granulomatous inflammation was characterised by infiltrates of epithelioid macrophages and multinucleated giant cells (Fig 2). These lymphoid tissues were depleted of mature lymphocytes and contained pyknotic basophilic nucleic and adjacent karyorrhectic debris; the germinal centres were reduced or absent. There was no significant difference between the mean (sd) lesion scores of groups $2(4.5[0.836])$ and $3(3.83$ [0.983]).

\section{In situ hybridisation}

Forty-two days after they were inoculated, PCV-2 DNA was detected in the lymph nodes of the pigs infected with PCV-2 (groups 1, 2 and 3) as distinct positive labelling scattered throughout the follicular areas. The distribution of positive cells was multifocal (Fig 3).

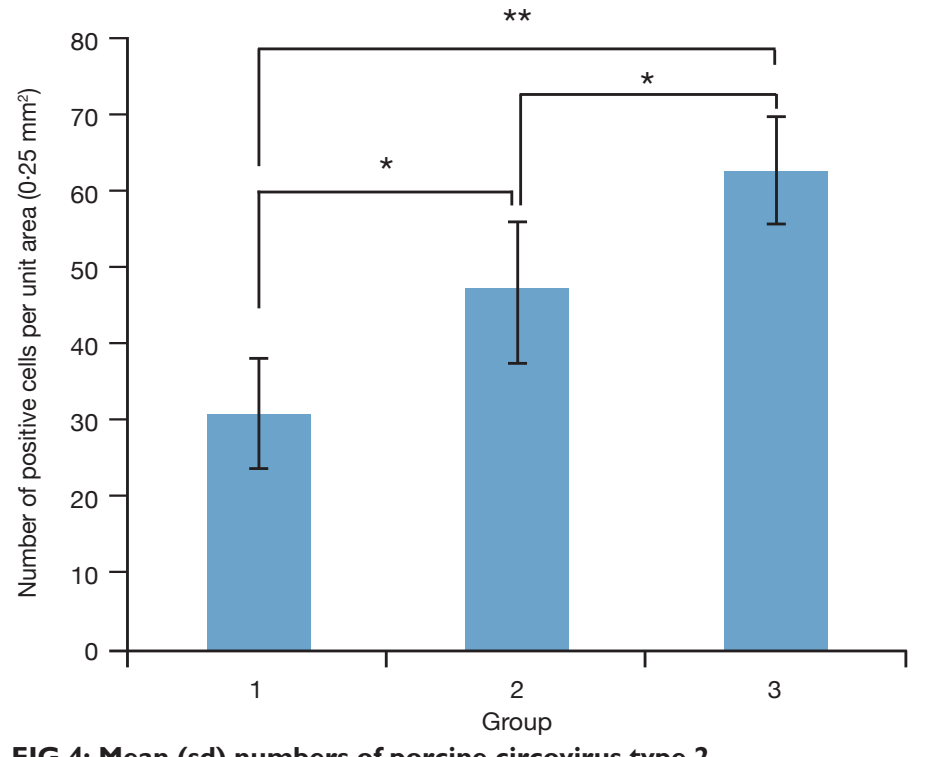

FIG 4: Mean (sd) numbers of porcine circovirus type 2 (PCV-2)-positive cells in the inguinal lymph nodes of pigs infected with PCV-2 (group I), infected with PCV-2 and immunised with modified classical swine fever virus (CSFV) vaccine (group 2), and co-infected with PCV-2 and porcine parvovirus (PPV) (group 3). $* \mathbf{P}<0.05$, ** $\mathbf{P}<0.001$

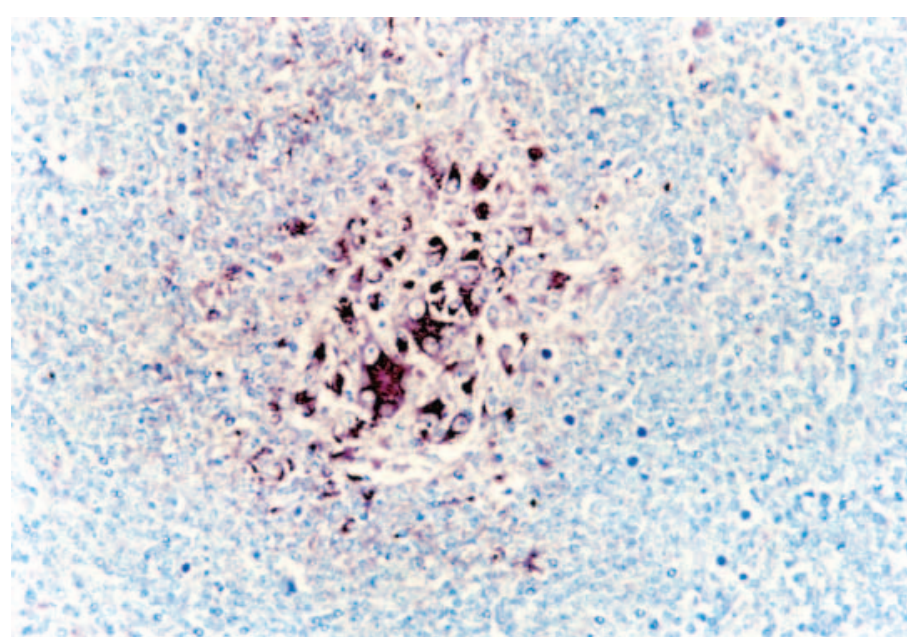

FIG 3: Porcine circovirus type 2 (PCV-2) DNA detected by in situ hybridisation in macrophages in an inguinal lymph node of a pig, which had been infected with PCV-2 and immunised with modified classical swine fever virus vaccine (group 2), and then euthanased 42 days after inoculation. DNA probe, nitroblue tetrazolium/ 5-bromocresyl-3-indolylphosphate, methyl green counterstain. x 400

In the superficial inguinal lymph node, the pigs in group 3 (PCV-2 plus PPV) had significantly more PCV-2-positive cells per unit area than the pigs in group 1 (PCV-2 only; $\mathrm{P}<0.001)$ and the pigs in group 2 ( $\mathrm{PCV}-2$ plus CSFV; $\mathrm{P}<0.05$ ), and the pigs in group 2 had significantly more PCV-2-positive cells than the pigs in group $1(\mathrm{P}<0.05)$ (Fig 4).

\section{Discussion}

The signs of PMWS were reproduced in conventional pigs by infecting them with PCV-2 and immunising them with modified live CSFV vaccine. Gross and histological lesions similar to those associated with PMWS had previously been induced by infecting pigs with PCV-2 and vaccinating them with Mycoplasma hyopneumoniae bacterins (Allan and others 2001, Kyriakis and others 2002, Opriessnig and others 2003, Krakowka and others 2007). However, a diagnosis of PMWS must meet three criteria: the presence of compatible clinical signs; the presence of characteristic microscopical lesions; and the presence of PCV-2 within these lesions. A positive result for only one of these three criteria is not diagnostic of PMWS, but because the pigs infected with PCV-2 and immunised with modified live CSFV vaccine satisfied all three criteria, they were diagnosed with PMWS.

The lymph nodes from the pigs infected with PCV-2 and immunised with modified live CSFV vaccine (group 2) contained more PCV-2-positive cells than the lymph nodes from the pigs infected with PCV-2 alone (group 1). This finding suggests that the activation of histiocytic lineage cells by immune stimulation with the modified live CSFV vaccine potentiates the replication of PCV-2 and the expression of the disease in the infected pigs. As a result, vaccinating commercial herds of pigs with the modified live CSFV vaccine might increase the incidence and severity of PMWS because there is a high incidence of subclinical PCV-2 infection in pig production facilities (Allan and Ellis 2000).

Although the CSFV vaccine potentiates the incidence of PMWS in pigs, there is no explanation for why its effect in the field was minimal or mild before vaccinations were stopped in Korea in 2001. The prevalence of PCV -2 infection between 1997 and 2000 might have been low, because PMWS was first reported in Korea in 1997 (Choi and others 2000). Alternatively, there could have been a change in the stage of PCV-2 infection in pigs before the vaccinations were stopped in 2001 and after they were reinstated in 2003. Immunostimulation after viraemia, when piglets have developed immunity, or immunostimulation before PCV-2 infection are unlikely to have the same effect as immunostimulation during an active viraemic PCV-2 infection (Opriessnig and others 2006, Krakowka and others 2007). These results provide valuable information for countries that may need to adopt emergency vaccination programmes to control outbreaks of CSF. Vaccination against CSFV is obligatory for all pigs in Korea, and it is therefore important 
that methods for minimising the potentiation of PMWS by CSFV vaccine should be investigated.

\section{Acknowledgements}

This research was supported by contract research funds from the Research Institute for Veterinary Science of the College of Veterinary Medicine, and by the Brain Korea 21 Programme for Veterinary Science in the Republic of Korea.

\section{References}

ANON (2001) 2000 report of the AVMA panel on euthanasia. Journal of the American Veterinary Medical Association 218, 669-696

ALLAN, G. M. \& ELLIS, J. A. (2000) Porcine circoviruses: a review. Journal of Veterinary Diagnostic Investigation 12, 3-14

Allan, G. M., KenNedy, S., MCNeilly, F., FOSter, J. C., Ellis, J. A. KRAKOWKA, S. J., MEEHAN, B. M. \& ADAIR, B. M. (1999) Experimental reproduction of severe wasting disease by co-infection of pigs with porcine circovirus and porcine parvovirus. Journal of Comparative Pathology 121, 1-11

ALLAN, G. M., MCNEILLY, F., ELLIS, J. A. \& KRAKOWKA, S. (2001) Neonatal vaccination for Mycoplasma hyopneumoniae and postweaning multisystemic wasting syndrome: a field trial. Pig Journal 48, 34-41

CHAE, C. (2004) Postweaning multisystemic wasting syndrome: a review of aetiology, diagnosis and pathology. Veterinary Journal 168, 41-49

CHOI, C., CHAE, C. \& CLARK, E. G. (2000) Porcine postweaning multisystemic wast ing syndrome in Korean pig: detection of porcine circovirus 2 infection by immunohistochemistry and polymerase chain reaction. Journal of Veterinary Diagnostic Investigation $12,151-153$

KIM, J. \& CHAE, C. (2004) Expression of monocyte chemoattractant protein-1 and macrophage inflammatory protein-1 in porcine circovirus 2 -induced granulomatous inflammation. Journal of Comparative Pathology 131, 121-126

KIM, J., CHOI, C. \& CHAE, C. (2003) Pathogenesis of postweaning multisystemic wasting syndrome reproduced by co-infection with Korean isolates of porcine circovirus 2 and porcine parvovirus. Journal of Comparative Pathology 128, 52-59 KRAKOWKA, S., ELLIS, J. A., MCNEILlY, F., RINGLER, S., RINGS, D. M. \& ALLAN, G. (2001) Activation of the immune system is the pivotal event in the production of wasting disease in pigs infected with porcine circovirus-2 (PCV-2). Veterinary Pathology 38, 31-42

KRAKOWKA, S., ELliS, J. A., MCNEILly, F., WALDNER, C., RINGS, D. M. \& ALLAN, G. (2007) Mycoplasma hyopneumoniae bacterins and porcine circovirus type 2 (PCV2) infection: induction of postweaning multisystemic wasting syndrome (PMWS) in the gnotobiotic swine model of PCV2-associated disease. Canadian Veterinary Journal 48, 716-724

KRAKOWKA, S., ELLIS, J. A., MEEHAN, B., KENNEDY, S., MCNEILLY, F. \& ALLAN, G. (2000) Viral wasting syndrome of swine: experimental reproduction of postweaning multisystemic wasting syndrome in gnotobiotic swine by coinfection with porcine circovirus 2 and porcine parvovirus. Veterinary Pathology 37, 254-263

KYRIAKIS, S. C., SAOULIDIS, K., LEKKAS, S., MILIOTIS, CH. C., PAPOUTSIS, P. A. \& KENNEDY, S. (2002) The effects of immuno-modulation on the clinical and pathological expression of postweaning multisystemic wasting syndrome. Journal of Comparative Pathology 126, 38-46

MOENNIG, V. (1992) The hog cholera virus. Comparative Immunology, Microbiology and Infectious Diseases 15, 189-201

OPRIESSNIG, T., HALBUR, P. G., YU, S., THACKER, E. L., FENAUX, M. \& MENG X. J. (2006) Effects of the timing of the administration of Mycoplasma hyopneumoniae bacterin on the development of lesions associated with porcine circovirus type 2 . Veterinary Record 158, 149-154

OPRIESSNIG, T., YU, S., GALLUP, J. M., EVANS, R. B., FENAUX, M., PALLARES, F. THACKER, E. L., BROCKUS, C. W. ACKERMANN, M. R., THOMAS, P., MENG, X. J. \& HALBUR, P. G. (2003) Effect of vaccination with selective bacterins on conventional pigs infected with type 2 porcine circovirus. Veterinary Pathology 40, 521-529

RESENDES, A., SEGAlÉS, J., BALASCH, M., CALSAMiglia, M., SiBIlA, M., ELLERBROK, H., MATEU, E., PLANA-DURÁN, J., MANKERTZ, A. \& DOMINGO, M. (2004) Lack of an effect of a commercial vaccine adjuvant on the development of postweaning multisystemic wasting syndrome (PMWS) in porcine circovirus type 2 (PCV2) experimentally infected conventional pigs. Veterinary Research 35, 83-90 